Pacific Journal of Mathematics

NONLINEAR ELLIPTIC CONVOLUTION EQUATIONS OF 


\section{NONLINEAR ELLIPTIC CONVOLUTION EQUATIONS OF WIENER-HOPF TYPE IN A BOUNDED REGION}

\section{BuI AN ToN}

The existence of a solution of a nonlinear perturbation of an elliptic convolution equation of Wiener-Hopf type in a bounded region $\mathbf{G}$ of $\mathbf{R}^{n}$ is proved. More explicitly, let $A$ be an elliptic convolution operator on $G$ of order $\alpha, \alpha>0 ; A_{j}$ the principal part of $A$ in a local coordinate system and $\tilde{A}_{j}\left(x^{j}, \xi\right)$ be the symbol of $A_{j}$ with a factorization with respect to $\xi_{n}$ of the form: $\widetilde{A}_{j}\left(x^{j}, \xi\right)=\widetilde{A}_{j}^{+}\left(x^{j}, \xi\right) \widetilde{A}_{j}^{-}\left(x^{j}, \xi\right)$ for $x_{n}^{j}=0 . \tilde{A}_{j}^{+}, \widetilde{A}_{j}^{\bar{j}}$ are homogeneous of orders $0, \alpha$ in $\xi$ respectively; the first admitting an analytic continuation in $\operatorname{Im} \xi_{n}>0$, the second in $\operatorname{Im} \xi_{n} \leqq 0$. Let $T_{k}, k=0, \cdots,[\alpha]-1$ be bounded linear operators from $H_{+}^{k}(G)$ into $L^{2}(G)$ where $H_{+}^{k}(G), k \geqq 0$ are the Sobolev-Slobo detskii spaces of generalized functions.

The purpose of the paper is to prove the solvability of: $A u_{+}+\lambda^{\alpha} u_{+}=f\left(x, T_{0} u_{+}, \cdots, T_{[\alpha]-1} u_{+}\right)$on $G ; u_{+}$in $H_{+}^{\alpha}(G)$ for large $|\lambda|$ and on a ray $\arg \lambda=\theta$ such that $\tilde{A}_{j}+\lambda^{\alpha} \neq 0$ for $|\xi|+|\lambda| \neq 0$ and for all $j . \quad f\left(x, \zeta_{0}, \cdots, \zeta_{\alpha-1}\right)$ has at most a linear growth in $\left(\zeta_{0}, \cdots, \zeta_{\alpha-1}\right)$ and is continuous in all the variables.

Linear elliptic convolution equations in a bounded region for arbitrary $\alpha$ and with symbols having the above type of factorization $(\lambda=0)$ have been considered recently by Visik-Eskin [3]. Those equations are similar to integral equations since no boundary conditions are required.

The notation and terminology are those of Visik-Eskin and are given in $\S 1$. The theorems are proved in $\S 2$.

1. Let $s$ be an arbitrary real number and $H^{s}\left(R^{n}\right)$ be the SobolevSlobodetskii space of (generalized) functions $f$ such that:

$$
\|f\|_{s}^{2}=\int_{E^{n}}\left(1+|\xi|^{2}\right)^{s}|\widetilde{f}(\xi)|^{2} d \xi<+\infty
$$

where $\tilde{f}(\xi)$ is the Fourier transform of $f$.

We denote by $H^{s}\left(R_{+}^{n}\right)$, the space consisting of functions defined on $R_{+}^{n}=\left\{x: x_{n}>0\right\}$ and which are the restrictions to $R_{+}^{n}$ of functions in $H^{s}\left(R^{n}\right)$. Let $l f$ be an extension of $f$ to $R^{n}$, then:

$$
\|f\|_{s}^{+}=\|f\|_{H^{s}\left(R_{+}^{n}\right)}=\inf \|l f\|_{s} .
$$

The infimum is taken over all extensions $l f$ of $f$.

The $\stackrel{\circ}{H}_{0}^{+}=\left\{f_{+} ; f_{+}(x)=f(x)\right.$ if $x_{n}>0, f \in L^{2}\left(R^{n}\right), f_{+}(x)=0$ if $\left.x_{n} \leqq 0\right\}$ 
and similarly for $\stackrel{\circ}{H}_{0}^{-}$.

We denote by $H_{s}^{+}$, the space of functions $f_{+}$with $f_{+}$in $\stackrel{\circ}{H}_{0}^{+}$and $f_{+} \in H^{s}\left(R_{+}^{n}\right)$ on $R_{+}^{n}$.

$\stackrel{\circ}{H}_{s}^{+}$is the subspace of $H^{s}\left(R^{n}\right)$ consisting of functions with supports in $\mathrm{cl}\left(R_{+}^{n}\right)$. $\widetilde{H}_{s}^{+}, \widetilde{H}_{s}, \widetilde{\stackrel{H}{H}}_{s}^{+}$denote respectively the spaces which are the Fourier images of $H_{s}^{+}, H^{s}\left(R^{n}\right), \stackrel{\circ}{H_{s}^{+}}$.

Let $\tilde{f}(\xi)$ be a smooth decreasing (i.e., $|\tilde{f}(\xi)| \leqq M\left|\xi_{n}\right|^{-1-\varepsilon}$ for large $\left|\xi_{n}\right|$ and for some $\varepsilon>0$ ) function. The operator $\Pi^{+}$is defined as:

$$
\Pi^{+} \widetilde{f}(\xi)=\frac{1}{2} \widetilde{f}(\xi)+i(2 \pi)^{-1} \text { v.p. } \int_{-\infty}^{\infty} \widetilde{f}\left(\xi^{\prime}, \eta_{n}\right)\left(\xi_{n}-\eta_{n}\right)^{-1} d \eta_{n}
$$

where $\xi^{\prime}=\left(\xi_{1}, \cdots, \xi_{n-1}\right)$.

For any $\widetilde{f}$, then the above relation is understood as the result of the closure of the operator $\Pi^{+}$defined on the set of smooth and decreasing functions.

$\Pi^{+}$is a bounded mapping from $\widetilde{H}_{s}$ into $\widetilde{\stackrel{H}{H}}_{s}^{+}$if $0 \leqq s<1 / 2$ and is a bounded mapping from $\widetilde{H}_{s}$ into $\widetilde{H}_{s}^{+}$if $s \geqq 1 / 2$.

Set: $\xi_{-}=\xi_{n}-i\left|\xi^{\prime}\right| ;\left(\xi_{-}-i\right)^{s}$ is analytic for any $s$ if $\operatorname{Im} \xi_{n} \leqq 0$ and:

$$
\|f\|_{s}^{+}=\left\|\Pi^{+}\left(\xi_{-}-i\right)^{s} l \widetilde{f}(\xi)\right\|_{0}
$$

where $l f$ is any extension of $f$ to $R^{n}$ (Cf. [3], p. 93 relation (8.1)).

Let $G$ be a bounded open set of $R^{n}$ with a smooth boundary. $H^{s}(G)$ denotes the restriction to $G$ of functions in $H^{s}\left(R^{n}\right)$ with the norm:

$$
\|u\|_{s}=\inf \|v\|_{H^{s}\left(R^{n}\right)} ; \quad v=u \text { on } G \text {. }
$$

By $H_{+}^{s}(G)$, we denote the space of functions $f$ defined on all of $R^{n}$, equal to 0 on $R^{n} / \mathrm{cl}(G)$ and coinciding in $\mathrm{cl} G$ with functions in $H^{s}(G)$.

DEFINITION 1. $\widetilde{A}(\xi)$ is in $0_{\alpha}$ if and only if:

(i) $\widetilde{A}(\xi)$ is a homogeneous function of order $\alpha$ in $\xi$.

(ii) $\widetilde{A}$ is continuous for $\xi \neq 0$.

Definition 2. $\widetilde{A}_{+}(\xi)$ is in $0_{\alpha}^{+}$if and only if:

(i) $\widetilde{A}_{+}(\xi)$ is in $0_{\alpha}$.

(ii) $\widetilde{A}_{+}\left(\xi^{\prime}, \xi_{n}\right)$ has an analytic continuation with respect to $\xi_{n}$ in the half-plane $\operatorname{Im} \xi_{n}>0$ for each $\xi^{\prime}$.

Similar definition for $0_{\alpha}^{-}$:

Definition 3. $\widetilde{A}$ is in $E_{\alpha}$ if and only if:

(i) $\widetilde{A}$ is in $0_{\alpha}$. 
(ii) $\widetilde{A}(\xi) \neq 0$ for $\xi \neq 0$.

(iii) $\widetilde{A}(\xi)$ has, for $\xi^{\prime} \neq 0$, continuous first order derivatives, bounded if $|\xi|=1, \xi^{\prime} \neq 0$.

Definition 4. $\widetilde{A}\left(x, \xi^{\prime}, \xi_{n}\right)$ is in $D_{\alpha}^{0}$ if and only if: $\xi \neq 0$.

(i) $\widetilde{A}(x, \xi)$ is infinitely differentiable with respect to $x$ and $\xi$;

(ii) $\widetilde{A}(x, \xi)$ is in $0_{\alpha}$ for $x$ in $R^{n}$.

(iii) $\quad a_{k 2}(x)=\frac{\partial^{k}}{\left(\partial \xi^{\prime}\right)^{k}} \widetilde{A}(x, 0,-1)=(-1)^{k} \exp (-i \alpha \pi) \frac{\partial^{k}}{\left(\partial \xi^{\prime}\right)^{k}} \widetilde{A}(x, 0,1)$ $x$ in $R^{n} ; 0 \leqq|k|<\infty ; k=\left(k_{1}, \cdots, k_{n}\right)$.

Definition 5. Let $A$ be a bounded linear operator from $H_{s}^{+}$into $H^{s-\alpha}\left(R_{+}^{n}\right)$. Then any bounded linear operator $T$ from $H_{s-1}^{+}$into $H^{s-\alpha}\left(R_{+}^{n}\right)$, (or from $H_{s}^{+}$into $H^{s-\alpha+1}\left(R_{+}^{n}\right)$ ) is called a right (left) smoothing operator with respect to $A$.

$T$ is a smoothing operator with respect to $A$ if it is both a left ane right smoothing operator.

Let $\widetilde{A}(\xi)$ be in $0_{\alpha}$ for $\alpha>0$. For $u_{+}$in $H_{s}^{+}, s \geqq 0$, with support in $\operatorname{cl}\left(R_{+}^{n}\right)$, set: $A u_{+}=F^{-1}\left(\widetilde{A}(\xi) \tilde{u}_{+}(\xi)\right)$ where $F^{-1}$ is the inverse Fourier transform. It is well defined in the sense of generalized functions. $A$ is a bounded linear operator from $H_{s}^{+}$into $H^{s-\alpha}\left(R^{n}\right)$.

Let $\widetilde{A}(x, \xi)$ be an element of $E_{\alpha}$ for each $x$ in $\operatorname{cl} G$ and $\widetilde{A}(x, \xi)$ be infinitely differentiable with respect to $x$ and $\xi$. Since $G$ is a bounded set of $R^{n}$, we may assume that $G$ is contained in a cube of side $2 p$ centered at 0 . We extend $\widetilde{A}(x, \xi)$ with respect to $x$ to all of $R^{n}$ by setting $\widetilde{A}(x, \xi)=0$ if $|x| \geqq p-\varepsilon$ for $\varepsilon>0$. We get a finite function, homogeneous of order $\alpha$ with respect to $\xi$.

We take the expansion into Fourier series of $\widetilde{A}(x, \xi)$ :

$$
\widetilde{A}(x, \xi)=\sum_{k=-\infty}^{\infty} \psi_{0}(x) \exp [(i \pi k x) / p] \widetilde{L}_{k}(\xi) ; \quad k=\left(k_{1}, \cdots, k_{n}\right)
$$

where:

$$
\widetilde{L}_{k}(\xi)=(2 p)^{-n} \int_{-p}^{p} \exp [(-i \pi k x) / p] \widetilde{A}(x, \xi) d x
$$

$\psi_{0}(x)=1$ for $|x| \leqq p-\varepsilon ; \psi_{0}(x)=0$ for $|x| \geqq p ; \psi_{0}(x) \in C_{c}^{\infty}\left(R^{n}\right)$. We have: $\left|\widetilde{L}_{k}(\xi)\right| \leqq C|\xi|^{\alpha}(1+|k|)^{-M}$ for arbitrary positive $M$. Let $u_{+}$be in $H_{+}^{s}(G)$, we define:

$$
A u_{+}=\sum_{-\infty}^{\infty} \psi_{0}(x)[\exp ((i k x \pi) / p)] L_{k} * u_{+}
$$

where $L_{k} * u_{+}=L_{k} u_{+}$is defined as before since $\widetilde{L}_{k}(\xi)$ is independent of $x$. 
Denote by $P^{+}$, the restriction operator of functions defined on $R^{n}$ to $G$. We consider an elliptic convolution equation of order $\alpha$, on $G$ of the form:

$$
P^{+} A u_{+}=\sum_{j} P^{+} \varphi_{j} A \psi_{j} u_{+}+T u_{+}
$$

$T$ is a smoothing operator. The $\varphi_{j}$ is a finite partition of unity corresponding to a covering $N_{j}$ of $\mathrm{cl} G$ with diam $\left(N_{j}\right)$ sufficiently small. The $\psi_{j}$ are in $C_{c}^{\infty}\left(R^{n}\right)$ with $\varphi_{j} \psi_{j}=\varphi_{j}$ and $\operatorname{supp}\left(\psi_{j}\right) \subseteq N_{j}$.

Suppose $\tilde{A} \in D_{\alpha}^{0}$, then the operator $\varphi_{j} A \psi_{j}$ taken in local coordinates may be written as:

$$
\varphi_{j} A \psi_{j}=\varphi_{j} A_{j} \psi_{j}+T_{j}
$$

where $A_{j}$ is a convolution operator of the form (1.1) and $T_{j}$ is a smoothing operator (Cf. [3] Appendix 2).

2. The main result of the paper is the following theorem:

THEOREM 1. Let $A$ be an elliptic convolution operator on $G$, of order $\alpha>0$, and of the form (1.2). Suppose that:

(i) $\widetilde{A}_{j}\left(x^{j}, \xi\right) \in E_{\alpha} \cap D_{\alpha}^{0}$.

(ii) $\widetilde{A}_{j}\left(x^{j}, \xi\right)$ has for $x_{n}^{j}=0$ a factorization of the form:

$$
\widetilde{A}_{j}\left(x^{j}, \xi\right)=\widetilde{A}_{j}^{+}\left(x^{j}, \xi\right) \widetilde{A}_{j}^{-}\left(x^{j}, \xi\right)
$$

where $\widetilde{A}_{j}^{+} \in 0_{0}^{+} ; \widetilde{A}_{\bar{j}}^{-} \in 0_{\alpha}^{-}$for all $x^{j} \in N_{j} \cap G$.

(iii) There exists a ray $\arg \lambda=\theta$ such that $\widetilde{A}_{j}\left(x^{j}, \xi\right)+\lambda^{\alpha} \neq 0$ for $|\xi|+|\lambda| \neq 0, x^{j} \in N_{j} \cap G$.

Let $f\left(x, \zeta_{0}, \cdots, \zeta_{[\alpha]-1}\right)$ be a function measurable in $x$ on $G$, continuous in all the other variables. Suppose there exists a positive constant $M$ such that:

$$
\left|f\left(x, \zeta_{0}, \cdots, \zeta_{[\alpha]-1}\right)\right| \leqq M\left\{1+\sum_{j=0}^{[\alpha]-1}\left|\zeta_{j}\right|\right\} .
$$

Let $T_{k} ; k=0, \cdots,[\alpha]-1$ be bounded, linear operators from $H_{+}^{k}(G)$ into $L^{2}(G)$. Then for $|\lambda| \geqq \lambda_{0}>0$; $\arg \lambda=\theta$; there exists a solution $u$ in $H_{+}^{\alpha}(G)$ of:

$$
P^{+}\left(A+\lambda^{\alpha}\right) u_{+}=f\left(x, T_{0} u_{+}, \cdots, T_{[\alpha]-1} u_{+}\right) \quad \text { on } G .
$$

The solution is unique if $f$ satisfies a Lipschitz condition in $\left(\zeta_{0}, \cdots, \zeta_{[\alpha]-1}\right)$.

To prove the theorem, we shall do as in [2]. First, following Visik-Agranovich [4], we establish an a priori estimate and show the existence and the uniqueness of a solution of a linear elliptic convolution 
equation depending on a large parameter in a bounded region. Then we use the Leray-Schauder fixed point theorem to prove Theorem 1 . We have:

THEOREM 2. Let $A$ be an elliptic convolution operator, of order $\alpha>0$, of the form (1.2). Suppose that all the hypotheses of Theorem 1 are satisfied. Let $f \in L^{2}(G)$; then there exists a unique solution $u_{+}$ in $H_{+}^{\alpha}(G)$ of:

$$
P^{+}\left(A+\lambda^{\alpha}\right) u_{+}=f \text { on } G ;|\lambda| \geqq \lambda_{0}>0 \quad \arg \lambda=\theta .
$$

Moreover:

$$
\left\|u_{+}\right\|_{\alpha}+|\lambda|^{\alpha}\left\|u_{+}\right\|_{0} \leqq M\|f\|_{0}
$$

where $M$ is independent of $\lambda, u_{+}$.

Proof of Theorem 1. Let $v$ be an element of $H_{+}^{\alpha}(G)$ and $0 \leqq t \leqq 1$. Consider the linear elliptic convolution equation:

$$
P^{+}\left(A u_{+}+\lambda^{\alpha} u_{+}\right)=f\left(x, t T_{0} v, \cdots, t T_{[\alpha]-1} v\right) .
$$

With the hypotheses of the theorem, $f\left(x, t T_{0} v, \cdots, t T_{[\alpha]-1} v\right)$ is in $L^{2}(\mathrm{G})$. It follows from Theorem 2 that there exists a unique solution $u_{+}$in $H_{+}^{\alpha}(G)$ of the problem.

Let $\mathscr{A}(t)$ be the nonlinear mapping from $[0,1] \times H_{+}^{\alpha}(G)$ into $H_{+}^{\alpha}(G)$ defined by $\mathscr{A}(t) v=u_{+}$where $u_{+}$is the unique solution of the above problem.

The theorem is proved if we can show that $\mathscr{A}(1)$ has a fixed point.

Proposition 1. $\mathscr{A}(t)$ is a completely continuous mapping from $[0,1] \times H_{+}^{\alpha}(G)$ into $H_{+}^{\alpha}(G)$.

Proof. (i) $\mathscr{A}(t)$ is continuous. Suppose that $t_{n} \rightarrow t ; t_{n}, t$ in $[0,1] v_{n} \rightarrow v$ in $H_{+}^{\alpha}(G)$. Set: $u_{n}=\mathscr{A}\left(t_{n}\right) v_{n}$. Then from Theorem 2, we get:

$$
\begin{aligned}
\left\|u_{n}-u\right\|_{\alpha} \leqq M \| f\left(\cdot, t_{n} T_{0} v_{n},\right. & \left.\cdots, t_{n} T_{[\alpha]-1} v_{n}\right) \\
& -f\left(\cdot, t T_{0} v, \cdots, t T_{[\alpha]-1} v\right) \|_{0} .
\end{aligned}
$$

It follows from Lemmas 3.1 and 3.2 of [1] that $u_{n} \rightarrow u$ in $H_{+}^{\alpha}(G)$.

(ii) $\mathscr{A}(t)$ is compact. Suppose that $\left\|v_{n}\right\|_{\alpha} \leqq M$. Then from the weak compactness of the unit ball in a Hilbert space and from the generalized Sobolev imbedding theorem, we get:

$$
v_{n_{j}} \rightarrow v \text { weakly in } H_{+}^{\alpha}(G) \text { and strongly in } H_{+}^{\alpha-\varepsilon}(G) ; 0<\varepsilon, \alpha-\varepsilon \geqq 0 \text {. }
$$


Applying the argument of the first part, we get the compactness of $\mathscr{A}(t)$.

Proposition 2. $I-\mathscr{A}(0)$ is a homeomorphism of $H_{+}^{\alpha}(G)$ into itself. If $v=\mathscr{A}(t) v$, for $0<t \leqq 1$; then: $\|v\|_{\alpha} \leqq M$ where $M$ is independent of $t$.

Proof. The first assertion is trivial.

Suppose that $v=\mathscr{A}(t) v$. It follows from Theorem 2 that:

$$
\begin{gathered}
\|v\|_{\alpha}+|\lambda|^{\alpha}\|v\|_{0} \leqq M\left\|f\left(\cdot, t T_{0} v, \cdots, t T_{[\alpha]-1} v\right)\right\|_{0} \\
\leqq M\left\{1+\|v\|_{[\alpha]-1}\right\} .
\end{gathered}
$$

It is well-known that:

$$
\|v\|_{[\alpha]-1} \leqq 1 / 2 M\|v\|_{\alpha}+C\|v\|_{0} \text {. }
$$

Taking $|\lambda|$ sufficiently large, we have: $\|v\|_{\alpha} \leqq M_{2} . \mathscr{A}(t)$ satisfies the hypotheses of the Leray-Schauder fixed point theorem (the uniform continutiy condition as in [2] is not necessary). So $\mathscr{A}(1)$ has a fixed point, i.e. $\mathscr{A}(1) u_{+}=u_{+}$.

The uniqueness of the solution in the case $f\left(x, \zeta_{0}, \cdots, \zeta_{[\alpha]-1}\right)$ satisfies a Lipschitz condition in $\left(\zeta_{0}, \cdots, \zeta_{[\alpha]-1}\right)$ follows trivially from the estimate of Theorem 2 . We shall not reproduce it.

Proof of Theorem 2. As usual, we consider first the case of the positive half-space $R_{+}^{n}$ with the convolution operator $A$ having a constant symbol.

Lemma 1. Let $\widetilde{A}(\xi)$ be an element of $E_{\alpha},(\alpha>0)$. Suppose that: $\widetilde{A}(\xi)=\widetilde{A}_{+}(\xi) \widetilde{A}_{-}(\xi)$ with $\widetilde{A}_{+}(\xi)$ in $0_{0}^{+}, \widetilde{A}_{-}(\xi)$ in $0_{\alpha}^{-}$. Let $P^{+}$be the restriction operator of functions in $R^{n}$ to $R_{+}^{n}$ and $A$ be the convolution operator with symbol $\widetilde{A}(\xi)$. Suppose there exists a ray $\arg \lambda=\theta$ such that: $\widetilde{A}(\xi)+\lambda^{\alpha} \neq 0$ for $|\xi|+|\lambda| \neq 0$. If $f$ is in $H^{0}\left(R_{+}^{n}\right)$, then there exists a unique solution $u$ in $H_{\alpha}^{+}$of:

$$
P^{+}\left(A+\lambda^{\alpha}\right) u_{+}=f \text { on } R_{+}^{n} ;|\lambda| \geqq \lambda_{0}>0 \text {. }
$$

Moreover:

$$
\left\|u_{+}\right\|_{\alpha}^{+}+|\lambda|^{\alpha}\left\|u_{+}\right\|_{0}^{+} \leqq M\|f\|_{0}^{+}
$$

where $M$ is independent of $\lambda, u_{+}, f$.

Proof. Set $\widetilde{A}(\xi, \lambda)=\widetilde{A}(\xi)+\lambda^{\alpha}$. It is homogeneous of order $\alpha$ in $(\xi, \lambda)$. Since $\widetilde{A}(\xi)$ is in $E_{\alpha}$, we have the following factorization with respect to $\xi_{n}$, which is unique up to a constant multiplier: 


$$
\widetilde{A}(\xi)=\widetilde{A}_{+}(\xi) \widetilde{A}_{-}(\xi)
$$

(Cf. Theorem 1.2 of [3], p. 95). The same proof with $\xi_{+}=\xi_{n}+i\left|\xi^{\prime}\right|$ replaced by $\xi_{+}^{2}=\xi_{n}+i\left(|\lambda|+\left|\xi^{\prime}\right|\right)$ and $\xi_{-}$replaced by:

$$
\xi_{-}^{\lambda}=\xi_{n}-i\left(|\lambda|+\left|\xi^{\prime}\right|\right)
$$

gives:

$$
\widetilde{A}(\xi, \lambda)=\widetilde{A}_{+}(\xi, \lambda) \widetilde{A}_{-}(\xi, \lambda)
$$

Moreover:

If $\widetilde{A}_{+}(\xi)$ is in $0_{0}^{+}$, then $\widetilde{A}_{+}(\xi, \lambda)$ is also in $O_{0}$ (is homogeneous of order 0 in $(\xi, \lambda))$. Similarly for $\tilde{A}_{-}(\xi, \lambda)$.

Let $l f(x)$ be an extension of $f$ to $R^{n}$. Consider:

$$
\tilde{u}_{+}(\xi)=\left(\tilde{A}_{+}(\xi, \lambda)\right)^{-1} \Pi^{+} l \tilde{f}(\xi)\left(\tilde{A}_{-}(\xi, \lambda)\right)^{-1} \text {. }
$$

For $|\lambda| \neq 0, \widetilde{u}_{+}(\xi)$ has an analytic continuation in $\operatorname{Im} \xi_{n}>0$ and:

$$
\int\left|\widetilde{u}_{+}\left(\xi^{\prime}, \xi_{n}+i \tau\right)\right|^{2} d \xi^{\prime} d \xi_{n} \leqq C,
$$

$C$ is independent of $\tau>0$. So: $\tilde{u}_{+}(\xi) \in \widetilde{\stackrel{\circ}{H}}_{0}^{+}$. (Cf. [3], p. 91).

We get:

$$
\begin{aligned}
\left\|u_{+}\right\|_{\alpha}^{+}= & \left\|\Pi^{+}\left(\xi_{-}-i\right)^{\alpha} \widetilde{u}_{+}(\xi)\right\|_{0}^{+} \\
& \leqq\left\|\left(\xi_{-}-i\right)^{\alpha}\left(\widetilde{A}_{+}(\xi, \lambda)\right)^{-1} \Pi^{+} l \tilde{f}(\xi)\left(\tilde{A}_{-}(\xi, \lambda)\right)^{-1}\right\|_{0} .
\end{aligned}
$$

Since $\widetilde{A}_{+}(\xi, \lambda)$ is homogeneous of order 0 in $(\xi, \lambda)$, we have:

$$
\widetilde{A}_{+}(\xi, \lambda)=\widetilde{A}_{+}(\xi /(|\xi|+|\lambda|), \lambda /(|\xi|+|\lambda|)) .
$$

Let $c=\operatorname{Min}\left|\widetilde{A}_{+}(\xi, \lambda)\right|$ for $|\xi|+|\lambda|=1, \arg \lambda=\theta$. Then $c>0$ and is independent of $\lambda$.

So:

$$
\begin{aligned}
\left\|u_{+}\right\|_{\alpha}^{+} & \leqq c^{-1}\left\|\left(\xi_{-}-i\right)^{\alpha} \Pi^{+} l \widetilde{f}(\xi)\left(\widetilde{A}_{-}(\xi, \lambda)\right)^{-1}\right\|_{0} \\
& \leqq C\left\|l \widetilde{f}(\xi)\left(\widetilde{A}_{-}(\xi, \lambda)\right)^{-1}\right\|_{\alpha} .
\end{aligned}
$$

We may write:

$$
\widetilde{A}_{-}(\xi, \lambda)=(|\xi|+|\lambda|)^{\alpha} \widetilde{A}_{-}(\xi /(|\xi|+|\lambda|), \lambda /(|\xi|+|\lambda|)) .
$$

Let $C=\operatorname{Min}\left|\tilde{A}_{-}(\xi, \lambda)\right|$ for $|\xi|+|\lambda|=1, \arg \lambda=\theta$. Then $C>0$ and is independent of $\lambda$.

We obtain:

$$
\left\|u_{+}\right\|_{\alpha}^{+} \leqq C\|l \widetilde{f}(\xi)\|_{0} \leqq C_{2}\|f\|_{0}^{+} .
$$

A similar argument gives: 


$$
\left\|u_{+}\right\|_{0}^{+} \leqq C|\lambda|^{-\alpha}\|f\|_{0}^{+}
$$

So:

$$
\left\|u_{+}\right\|_{\alpha}^{+}+|\lambda|^{\alpha}\left\|u_{+}\right\|_{0}^{+} \leqq C\|f\|_{0}^{+} .
$$

$C$ is independent of $\lambda, f, u_{+}$.

A direct verification shows that $u_{+}$is a solution of the equation. It remains to show that the solution is unique. Let $v_{+}$be an element of $H_{\alpha}^{+}$. Suppose that $v_{+}$is also a solution of the equation. Then as in [3], $\widetilde{v}_{+}(\xi)$, its Fourier transform is given by an expression of the same form as $\tilde{u}_{+}(\xi)$ with $\tilde{l f}(\xi)$ replaced by $\widetilde{l_{1} f}(\xi)$. $l_{1} f$ being an extension of $f$ to $R^{n}$.

Set $l_{2} f=l f-l_{1} f$. Then $l_{2} f \in H_{0}^{-}$, so $\widetilde{l_{2} f} \in \widetilde{\tilde{o}_{0}^{-}}$. $\widetilde{l_{2} f}(\xi)(\widetilde{A}-(\xi, \lambda))^{-1}$ is analytic in $\operatorname{Im} \xi_{n} \leqq 0$ for $|\lambda| \neq 0$ and moreover:

$$
\int\left|\widetilde{l_{2} f}\left(\xi^{\prime}, \xi_{n}+i \tau\right)\right|^{2}\left|\widetilde{A}_{-}\left(\xi^{\prime}, \xi_{n}+i \tau\right)\right|^{-2} d \xi^{\prime} d \xi_{n} \leqq C
$$

where $C$ is independent of $\tau \leqq 0$.

Hence $\widetilde{l_{2} f}(\xi)\left(\widetilde{A}_{-}(\xi, \lambda)\right)^{-1}$ is in $\stackrel{\widetilde{o}}{H_{0}^{-}}$(Cf. [3], p. 91), so:

$$
\left.\left.\Pi^{+} \widetilde{l_{2} f(\xi)}(\widetilde{A}-(\xi, \lambda))^{-1}\right)\right)=0 .
$$

Therefore: $\widetilde{A}_{+}(\xi, \lambda)\left(\widetilde{u}_{+}(\xi)-\widetilde{v}_{+}(\xi)\right)=0$.

But $\widetilde{A}_{+}(\xi, \lambda) \neq 0$ for $|\lambda| \neq 0$, we get $\widetilde{u}_{+}=\widetilde{v}_{+}$Q.E.D.

Set:

$$
\begin{aligned}
& A_{1} u=\sum_{k=-\infty}^{\infty} \psi_{0}(x) \exp [(i k \pi x) / p] L_{k} * u \\
& A_{0} u=\sum_{k=-\infty}^{\infty} \psi_{0}\left(x_{0}\right) \exp [(i k \pi) / p] L_{k} * u
\end{aligned}
$$

where $L_{k}, \psi_{0}$ are as in $\S 1$.

LEMMA 2. Let $A_{1}, A_{0}$ be as above and $\psi(x)$ be in $C_{c}^{\infty}\left(R^{n}\right)$ with $\psi(x)=0$ for $\left|x-x_{0}\right|>\delta ;|\psi(x)| \leqq K$ where $K$ is independent of $\delta$. Then:

$$
\left\|\psi\left(A_{1}-A_{0}\right) u\right\|_{s-\alpha}^{+} \leqq C \delta\|u\|_{s}^{+}+C(\delta)\|u\|_{s-1}^{+}
$$

for all $u$ in $H_{s}^{+}, s \geqq 0$.

Proof. Cf. Lemma 4.7 of [3], p. 119.

Proof of Theorem 2 (continued). (1) First, we establish an $a$ priori estimate of the solutions. 
Consider:

$$
P^{+} \varphi_{j} A \psi_{j} u_{+}+\lambda^{\alpha} P^{+}\left(\varphi_{j} u_{+}\right)=P^{+}\left(\varphi_{j} f\right)-T u_{+}
$$

where $T$ is a smoothing operator with respect to $\varphi_{j} A \psi_{j}$.

It has been shown in [3] (Appendix 2) that in a local coordinates system, the operator $\varphi_{j} A \psi_{j}$ becomes: $\varphi_{j} A_{j} \psi_{j}+T_{j}$ where $A_{j}$ has for symbol $\widetilde{A}_{j}\left(x^{j}, \xi\right)$ and $T_{j}$ is a smoothing operator.

So, we have:

$$
P^{+} \varphi_{j} A_{j}\left(\psi_{j} u_{+}\right)+\lambda^{\alpha} P^{+}\left(\varphi_{j} u_{+}\right)=P^{+}\left(\varphi_{j} f\right)+T_{j}^{2} u_{+}
$$

where $T_{j}^{2}$ is again a smoothing operator.

Let $A_{j 0}$ be the convolution operator with symbol $\widetilde{A}_{j}\left(x_{0}^{j}, \xi\right)$ evaluted at the point $x_{0}^{j}$. We write:

$$
\begin{aligned}
P^{+} \varphi_{j} A_{j 0}\left(\psi_{j} u_{+}\right)+\lambda^{\alpha} P^{+}\left(\varphi_{j} u_{+}\right) & =P^{+}\left(\varphi_{j} f\right) \\
& +T_{j}^{2} u_{+}+P^{+} \varphi_{j}\left(A_{j 0}-A_{j}\right) \psi_{j} u^{+} .
\end{aligned}
$$

Applying Lemma 4.D.1 of [3] (p. 145), we have:

$$
P^{+} \varphi_{j} A_{j 0}\left(\psi_{j} u_{+}\right)=P^{+} A_{j 0}\left(\varphi_{j} u_{+}\right)+T_{j}^{3} u_{+}
$$

where $T_{j}^{3}$ is a smoothing operator.

Therefore:

$$
\left(A_{j 0}+\lambda^{\alpha}\right) \varphi_{j} u_{+}=\varphi_{j} f+T_{j}^{4} u_{+}+\varphi_{j}\left(A_{j 0}-A_{j}\right)\left(\psi_{j} u_{+}\right) .
$$

The symbols $\widetilde{A}_{j 0}$ satisfy the hypotheses of Lemma 1. Applying Lemma 1; 2, we obtain:

$$
\begin{aligned}
\left\|\varphi_{j} u_{+}\right\|_{\alpha}^{+}+|\lambda|^{\alpha}\left\|\varphi_{j} u_{+}\right\|_{0}^{+} \leqq M\left\{\left\|\varphi_{j} f\right\|_{0}^{+}+\left\|u_{+}\right\|_{0}\right. \\
\left.+1 / 2 M\left\|u_{+}\right\|_{\alpha}+\left\|\psi_{j} u_{+}\right\|_{\alpha}^{+}+\left\|\varphi_{j} u_{+}\right\|_{0}^{+}\right\}
\end{aligned}
$$

where we have used the well-known inequality:

$$
\left\|u_{+}\right\|_{\alpha-1} \leqq \varepsilon\left\|u_{+}\right\|_{\alpha}+C(\varepsilon)\left\|u_{+}\right\|_{0} \text {. }
$$

On the other hand: $\left\|\psi_{j} u_{+}\right\|_{\alpha}^{+} \leqq M\left\|u_{+}\right\|_{\alpha}$. Summing with respect to $j$, we get:

$$
\begin{aligned}
\left\|u_{+}\right\|_{\alpha} & +|\lambda|^{\alpha}\left\|u_{+}\right\|_{0} \leqq M\left\{\|f\|_{0}+1 / 2 M\left\|u_{+}\right\|_{\alpha}\right. \\
& \left.+\delta\left\|u_{+}\right\|_{\alpha}+K\left\|u_{+}\right\|_{0}\right\}
\end{aligned}
$$

Taking $\delta$ small and $|\lambda|$ sufficiently large, we have:

$$
\left\|u_{+}\right\|_{\alpha}+|\lambda|^{\alpha}\left\|u_{+}\right\|_{0} \leqq M\|f\|_{0} \text {. }
$$

So, if there exists a solution, then the solution is unique. 
(2) It remains to show the existence of a solution. From Lemma 1, we know that $P^{+}\left(A_{j 0}+\lambda^{\alpha}\right)$ has an inverse $R_{j 0}$. Let $\hat{R}_{j 0}$ be the operator $R_{j 0}$ expressed in the global system of coordinates of $G$. Consider:

$$
R f=\sum_{j} \varphi_{j} \hat{R}_{j 0}\left(\psi_{j} f\right) \text {. }
$$

$R$ is a bounded linear mapping from $L^{2}(G)$ into $H_{+}^{\alpha}(G)$.

We show that: $\mathscr{A} R f=P^{+}\left(A+\lambda^{\alpha}\right) R f=f+\mathscr{C} f$ with $\|\mathscr{C}\| \leqq 1 / 2$.

We have:

$$
\mathscr{A} R f=\sum_{j} P^{+}\left(A+\lambda^{\alpha}\right) \varphi_{j} \psi_{j} \hat{R}_{j 0}\left(\psi_{j} f\right)
$$

Applying Lemma 4.D.1. of [3], we may write:

$$
\mathscr{A} R f=\sum_{j} P^{+} \varphi_{j}\left(A+\lambda^{\alpha}\right) \psi_{j} \hat{R}_{j 0}\left(\psi_{j} f\right)+T R f
$$

where $T$ is a smoothing operator.

We express $\varphi_{j}\left(A+\lambda^{\alpha}\right) \psi_{j} \hat{R}_{j 0}\left(\psi_{j} f\right)$ in local coordinates. We get:

$$
\varphi_{j}\left(A_{j 0}+\lambda^{\alpha}\right) \psi_{j} R_{j 0}\left(\psi_{j} f\right)+\varphi_{j}\left(A_{j}-A_{j 0}\right) \psi_{j} R_{j 0}\left(\psi_{j} f\right)+T_{j}^{1} R_{j 0}\left(\psi_{j} f\right) \text {. }
$$

Using Lemma 4.D.1 of [3] again, we obtain:

$$
\begin{aligned}
& \varphi_{j}\left(A_{j 0}+\lambda^{\alpha}\right) R_{j 0}\left(\psi_{j} f\right)+\varphi_{j}\left(A_{j}-A_{j 0}\right) \psi_{j} R_{j 0}\left(\psi_{j} f\right)+T_{j}^{2} R_{j 0}\left(\psi_{j} f\right) \\
= & T_{j}^{2} R_{j 0}\left(\psi_{j} f\right)+\varphi_{j} f+\varphi_{j}\left(A_{j}-A_{j 0}\right) \psi_{j} R_{j 0}\left(\psi_{j} f\right)=\varphi_{j} f+\mathscr{C}_{j}\left(\psi_{j} f\right) .
\end{aligned}
$$

The $T_{j}$ are all smoothing operators.

Applying Lemma 1, we have:

$$
\left\|T_{j}^{2} R_{j 0}\left(\psi_{j} f\right)\right\|_{0}^{+} \leqq C\left\|R_{j 0}\left(\psi_{j} f\right)\right\|_{\alpha-1}^{+} \leqq \varepsilon\|f\|_{0}+C|\lambda|^{-\alpha}\|f\|_{0} .
$$

From Lemmas 1 and 2, we get:

$$
\begin{aligned}
\left\|\varphi_{j}\left(A_{j}-A_{j 0}\right) \psi_{j} R_{j 0}\left(\psi_{j} f\right)\right\|_{0}^{+} \leqq & \delta\left\|\psi_{j} R_{j 0}\left(\psi_{j} f\right)\right\|_{\alpha}^{+} \\
& +C(\delta)\left\|\psi_{j} R_{j 0}\left(\psi_{j} f\right)\right\|_{\alpha-1}^{+} \\
\leqq & \delta\|f\|_{0}+C(\delta)\left\|\hat{R}_{j 0}\left(\psi_{j} f\right)\right\|_{\alpha-1} \\
\leqq & \delta\|f\|_{0}+\varepsilon C(\delta)\left\|R_{j 0}\left(\psi_{j} f\right)\right\|_{\alpha} \\
& +C(\delta) M(\varepsilon)\left\|\hat{R}_{j 0}\left(\psi_{j} f\right)\right\|_{0} \\
\leqq & \{\delta+\varepsilon C(\delta)\}\|f\|_{0} \\
& +|\lambda|^{-\alpha} M(\varepsilon) C(\delta)\|f\|_{0} .
\end{aligned}
$$

Taking $\varepsilon, \delta$ small, $|\lambda|$ large enough, we have:

$$
\left\|\mathscr{C}_{j}\left(\psi_{j} f\right)\right\|_{0}^{+} \leqq \frac{1}{4 N}\|f\|_{0} .
$$

We obtain: 


$$
R f=f+T R f+\sum_{j} \hat{\mathscr{C}}_{j}\left(\psi_{j} f\right)=f+\mathscr{C} f
$$

where $\hat{\mathscr{C}}_{j}$ is the operator $\mathscr{C}_{j}$ expressed in the global coordinates system of $G$. We obtain: $\|\mathscr{C} f\|_{0} \leqq 1 / 4\|f\|_{0}+1 / 4\|f\|_{0}$ for large $|\lambda|$. Hence $\|\mathscr{C}\| \leqq 1 / 2$; therefore $(I+\mathscr{C})^{-1}$ exists. We define:

$$
\mathscr{A}^{-1}=R(I+\mathscr{C})^{-1} \text {. }
$$

The writer wishes to thank the referee for his remarks.

\section{BIBLIOGRAPHY}

1. F. E. Browder, Nonlinear elliptic boundary value problems II, Trans. Amer. Math. Soc. 117 (1965), 530-550.

2. B. A. Ton, On nonlinear elliptic boundary value problems, Bull. Amer. Math. Soc. 72 (1966), 307-313.

3. M. I. Visik and G. I. Eskin, Equations in convolution in a bounded region, Uspehi Mat. Nauk. 20 (1965), No. 3, 90-153; Russian Math. Surveys 20 (1965), 85-157.

4. M. I. Visik and M. S. Agranovich, Elliptic problems with a parameter and parabolic problems of general type, Uspehi Mat. Nauk. 19 (1964), 53-161; Russian Math. Surveys, No. 3 (1964), 53-157.

Received March 20, 1967.

UNIVERSity OF BRITISH COLUMBIA

VANCOUVER, CANADA 



\section{PACIFIC JOURNAL OF MATHEMATICS}

\section{EDITORS}

\author{
H. ROYDEN \\ Stanford University \\ Stanford, California
}

\author{
J. P. JANS \\ University of Washington \\ Seattle, Washington 98105
}

J. DugundJI

Department of Mathematics

Rice University

Houston, Texas 77001

RICHARD ARENS

University of California

Los Angeles, California 90024

\section{ASSOCIATE EDITORS}
E. F. BECKENBACH
B. H. NeumanN
F. WOLF
K. YOSIDA

\section{SUPPORTING INSTITUTIONS}

\author{
UNIVERSITY OF BRITISH COLUMBIA \\ CALIFORNIA INSTITUTE OF TECHNOLOGY \\ UNIVERSITY OF CALIFORNIA \\ MONTANA STATE UNIVERSITY \\ UNIVERSITY OF NEVADA \\ NEW MEXICO STATE UNIVERSITY \\ OREGON STATE UNIVERSITY \\ UNIVERSITY OF OREGON \\ OSAKA UNIVERSITY \\ UNIVERSITY OF SOUTHERN CALIFORNIA
}

\author{
STANFORD UNIVERSITY \\ UNIVERSITY OF TOKYO \\ UNIVERSITY OF UTAH \\ WASHINGTON STATE UNIVERSITY \\ UNIVERSITY OF WASHINGTON \\ AMERICAN MATHEMATICAL SOCIETY \\ CHEVRON RESEARCH CORPORATION \\ TRW SYSTEMS \\ NAVAL ORDNANCE TEST STATION
}

\footnotetext{
Mathematical papers intended for publication in the Pacific Journal of Mathematics should be in typed form or offset-reproduced, double spaced with large margins. Underline Greek letters in red, German in green, and script in blue. The first paragraph or two must be capable of being used separately as a synopsis of the entire paper. It should not contain references to the bibliography. Manuscripts may be sent to any one of the four editors. All other communications to the editors should be addressed to the managing editor, Richard Arens, University of California, Los Angeles, California 90024.

Each author of each article receives 50 reprints free of charge; additional copies may be obtained at cost in multiples of 50 .

The Pacific Journal of Mathematics is published monthly. Effective with Volume 16 the price per volume (3 numbers) is $\$ 8.00$; single issues, $\$ 3.00$. Special price for current issues to individual faculty members of supporting institutions and to individual members of the American Mathematical Society: $\$ 4.00$ per volume; single issues $\$ 1.50$. Back numbers are available.

Subscriptions, orders for back numbers, and changes of address should be sent to Pacific Journal of Mathematics, 103 Highland Boulevard, Berkeley 8, California.

Printed at Kokusai Bunken Insatsusha (International Academic Printing Co., Ltd.), 7-17, Fujimi 2-chome, Chiyoda-ku, Tokyo, Japan.

PUBLISHED BY PACIFIC JOURNAL OF MATHEMATICS, A NON-PROFIT CORPORATION

The Supporting Institutions listed above contribute to the cost of publication of this Journal, but they are not owners of publishers and have no responsibility for its content or policies.
} 


\section{Pacific Journal of Mathematics}

\section{Vol. 24, No. $3 \quad$ July, 1968}

Duane W. Bailey, On symmetry in certain group algebras ............ 413

Lawrence Peter Belluce and Surender Kumar Jain, Prime rings with a one-sided ideal satisfying a polynomial identity ................ 421

L. Carlitz, A note on certain biorthogonal polynomials ............. 425

Charles O. Christenson and Richard Paul Osborne, Pointlike subsets of a manifold ......................................... 431

Russell James Egbert, Products and quotients of probabilistic metric

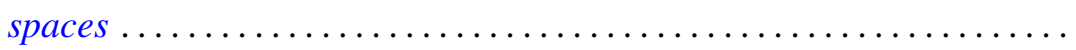

Moses Glasner, Richard Emanuel Katz and Mitsuru Nakai, Bisection into small annuli ..................................... 457

Karl Edwin Gustafson, A note on left multiplication of semigroup

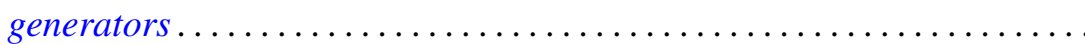

I. Martin (Irving) Isaacs and Donald Steven Passman, A characterization of groups in terms of the degrees of their characters. II ............. 467

Howard Wilson Lambert and Richard Benjamin Sher, Point-like

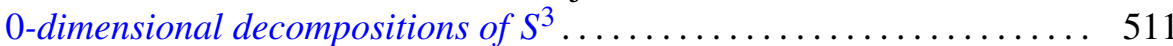

Oscar Tivis Nelson, Subdirect decompositions of lattices of width two ..... 519

Ralph Tyrrell Rockafellar, Integrals which are convex functionals . . . . . . . 525

James McLean Sloss, Reflection laws of systems of second order elliptic differential equations in two independent variables with constant coefficients ...

Bui An Ton, Nonlinear elliptic convolution equations of Wiener-Hopf type in a bounded region

Daniel Eliot Wulbert, Some complemented function spaces in $C(X)$

Zvi Ziegler, On the characterization of measures of the cone dual to a generalized convexity cone. 